\title{
Electron beam melting toward defects-free titanium alloys
}

\author{
H. Funagane *, Y.Takeda, M.Wada, H.Mizukami and S.Umeda \\ Nippon Steel Corporation \\ *Corresponding author: Email: funagane.zq3.hitoshi@jp.nipponsteel.com
}

\begin{abstract}
$\underline{\text { Abstract }}$
Titanium alloys used for rotating parts of aeroplanes must meet the strict standards of quality, so that it is required to demonstrate that electron beam melting is capable of producing defects-free ingots. However, complete elimination by dissolution of low density inclusions (LDI) having high nitrogen content would be difficult, so that a new way of eliminating LDI was needed. Therefore, control of a state of the molten titanium flow with the aide of Marangoni flow induced by electron beam has been studied, and the effects on eliminating LDI have been verified.
\end{abstract}

\section{Introduction}

Since it was introduced in 1980s, electron beam cold hearth melting [1] has been used to produce not only commercially pure titanium products but also aerospace-quality products. Among of them, titanium alloys used for rotating parts of aeroplanes must meet strict standards of quality, so that it is required to demonstrate that electron beam melting is capable of producing defectsfree ingots.

There are two kinds of inclusions (see table 1) to be eliminated. One is high density inclusions (HDI) such as WC tool bits and other refractory metals. The other is low density inclusions (LDI) such as nitrided sponge particles and burned sponge particles. Since density of HDI is greater than that of molten titanium (e.g., Ti-6Al-4V $\fallingdotseq 3920\left[\mathrm{~kg} / \mathrm{m}^{3}\right]$ ), they can sink in the molten titanium (pool) and can be trapped in the skull (solidified titanium formed on a crucible). On the other hand, LDI can have density lower than molten titanium, so that they do not sink but can move inside or on the surface of the pool. In order to eliminate those kinds of LDI, removal by dissolution is often used, but complete removal of LDI having high nitrogen content might be impossible [2, 3]. Therefore, a new way of eliminating LDI has been studied.

Table 1 Type of inclusions

\begin{tabular}{|c|c|c|c|}
\hline Type & Kind of inclusion & Density $\left[\mathrm{kg} / \mathrm{m}^{3}\right]$ & Melting point $\left[{ }^{\circ} \mathrm{C}\right]$ \\
\hline \multirow{2}{*}{ HDI } & WC tool bits & 15630 & 2870 \\
\cline { 2 - 4 } & A piece of W & 19250 & 3422 \\
\hline \multirow{2}{*}{ LDI } & Nitrided sponge particles & Irregular & $1670(0 \% \mathrm{~N})-$ \\
& & (True density 5400$)$ & $3000(15 \% \mathrm{~N})$ \\
\cline { 2 - 4 } & Burned sponge particles & Irregular & 1670 \\
\hline
\end{tabular}

\section{Methodology}

It is well known that there is a temperature gradient on the surface of a fluid, and thus Marangoni flow is induced. Therefore, some area of a molten titanium in a hearth is intensively heated by electron beam (hereafter, we call Intensive Heating (IH)), and thus Marangoni flow originating the heated area is generated. Based on the fact, we considered the control of a state of the molten titanium flow with the aide of the Marangoni flow, and inclusions coming to the area got stuck in front of the area (see figure 1). 


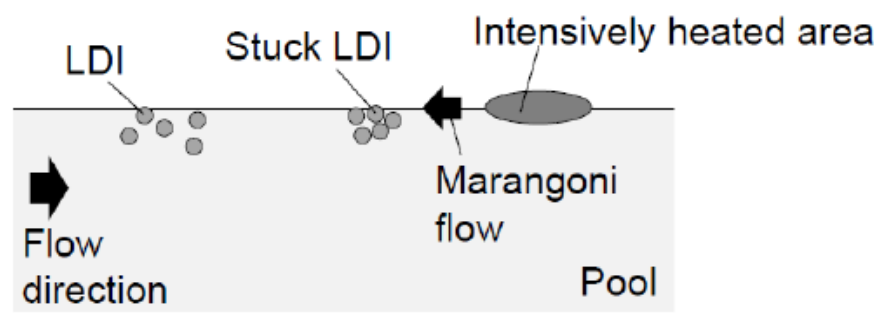

Figure 1 Mechanism of removing LDI

\section{Verification of effectiveness of IH by numerical simulation}

In order to verify that Intensive Heating can really get LDI stuck in front of the heated area, a computational model describing a molten titanium flow and behaviour of inclusions in a molten titanium has been developed [4-6].

\subsection{Computational model}

Equations describing molten metal flow are shown:

$$
\begin{aligned}
& \frac{\partial \rho}{\partial t}+\nabla \cdot(\rho \vec{v})=0 \\
& \frac{\partial}{\partial t}(\rho \vec{v})+\nabla \cdot(\rho \vec{v} \vec{v})=-\nabla p+\nabla \cdot \overline{\bar{\tau}}+\rho \vec{g}-\rho \beta^{\prime}\left(T-T_{0}\right) \vec{g}+\frac{(1-\beta)^{2}}{\beta^{3}} A_{\operatorname{mesh}} \vec{v} \\
& \overline{\bar{\tau}}=\mu\left(\nabla \vec{v}+\nabla \vec{v}^{\mathrm{T}}-\frac{2}{3} \nabla \cdot \vec{v} I\right) \\
& \frac{\partial}{\partial \mathrm{t}}(\rho H)+\nabla \cdot(\rho \vec{v} H)=\nabla \cdot(\lambda \nabla T) \\
& H=c_{P} T+\beta L \\
& \left\{\begin{array}{cl}
\beta=0 & \text { if } T<T_{\text {Solid }} \\
\beta=1 & \text { if } T>T_{\text {Liquid }} \\
\beta=\frac{T-T_{\text {Solid }}}{T_{\text {Liquid }}-T_{\text {Solid }}} & \text { if } T_{\text {Solid }}<T<T_{\text {Liquid }}
\end{array}\right.
\end{aligned}
$$

Here, $t$ is time, $\rho$ is density, $\vec{v}$ is velocity vector, $p$ is pressure, $\overline{\bar{\tau}}$ is stress tensor, $\mu$ is viscosity, $I$ is unit $\vec{g}$ is gravitational acceleration, $\beta^{\prime}\left(=1.73 \cdot 10^{-4}[1 / \mathrm{K}]\right)$ is thermal expansion coefficient, $T_{0}(=1923[\mathrm{~K}])$ is re temperature, $\beta$ is liquid fraction, $A_{\text {mesh }}=10^{5}, H$ is enthalpy, $\lambda$ is thermal conductivity, $T$ is temperature specific heat, $L(=286[\mathrm{~kJ} / \mathrm{kg}])$ is latent heat, $T_{\text {Solid }}(=1913[\mathrm{~K}])$ is solidus temperature, $T_{\text {Liquid }}(=1923$ liquidus temperature. The values of $c_{P}$ and $\lambda$ are summarized in Appendix A. 
Equations describing the behaviour of inclusions in a fluid are shown:

$$
\begin{gathered}
\frac{d \vec{u}_{P}}{d t}=\frac{\vec{v}-\vec{u}_{P}}{\tau_{r}}+\frac{\vec{g}\left(\rho_{P}-\rho\right)}{\rho_{P}} \\
\tau_{r}=\frac{\rho_{P} d_{P}^{2}}{18 \mu} \frac{24}{C_{d} \operatorname{Re}} \\
\operatorname{Re}=\frac{\rho d_{P}\left|\vec{u}_{P}-\vec{u}\right|}{\mu}
\end{gathered}
$$

Here, the first term of the right hand side of (7) represents drag force. $\tau_{\mathrm{r}}$ is relaxation time, $\vec{u}_{P}$ is particle (inc] velocity vector, $\rho_{P}$ is particle density, $d_{P}$ is particle diameter, $C_{d}$ is drag force coefficient [7], Re is $\mathrm{r}$ Reynolds number defined by equation (9).

Moreover, heat flux given to the surface of molten titanium is described by the following equations:

$$
\begin{gathered}
\mathrm{q}=\alpha q_{0} \exp \left(-\frac{\left(x-x_{0}\right)^{2}+\left(y-y_{0}\right)^{2}}{2 \sigma^{2}}\right)-C_{1} C_{2}\left(T^{4}-T_{\infty}^{4}\right) \\
\mathrm{Q}=\alpha q_{0} \int_{\substack{\text { all } x \\
\text { all }}} \exp \left(-\frac{\left(x-x_{0}\right)^{2}+\left(y-y_{0}\right)^{2}}{2 \sigma^{2}}\right) d x d y
\end{gathered}
$$

Here, q is heat flux given to the pool surface. The first term of (10) is heat flux by electron beam and the second 1 heat flux by radiation. $\alpha(=0.7)$ is coefficient representing heat efficiency, $(x, y)$ represents the position on th surface, $\left(x_{0}, y_{0}\right)$ is beam spot, $\sigma(=7.5[\mathrm{~mm}])$ is standard deviation, $C_{1}(=0.4)$ is external emissivity, $C_{2}(=$ $\left.10^{-8}\left[\mathrm{~W} / \mathrm{m}^{2} \mathrm{~K}^{4}\right]\right)$ is Stefan-Boltzmann constant, $T_{\infty}$ is external radiation temperature, $Q$ is heat of electron bean

\subsection{Numerical Conditions}

Flow of a molten titanium and behaviour of LDI moving on the surface of the pool was investigated under the following numerical conditions:

n Electron beam

- Heat, Scan velocity: 0.25 [MW], $1.6[\mathrm{~m} / \mathrm{s}]$

n LDI

- Diameter, Density:5.0 [mm], 3528 [kg/m3] (c.f., Molten Ti-6Al-4V: 3920 [kg/m3])

- Nitrogen content: $\infty$ (It was assumed that LDI did not dissolve.)

\subsection{Numerical Results}

Behaviour of LDI without Intensive Heating is shown in figure 2. In the figure, molten titanium flows from the left to the right, so that the solid lines in figure 2(b) representing LDI path on the surface of the pool are reaching to the lip where refined molten titanium is poured to a mold. This result indicates that LDI cannot be eliminated, and three is a risk that LDI contaminate ingots. 
On the other hand, behavior of LDI with IH is shown in figure 3. In the calculation, IH was carried out at the center of the hearth, so that the surface temperature becomes greater than other. Because of this temperature gradient, Marangoni flow is induced and LDI coming from the left get stuck in front of the hotter area and cannot move to the lip. This result does not change even if the position of beam spot changes as shown in state 1 to 3 in figure 3 .

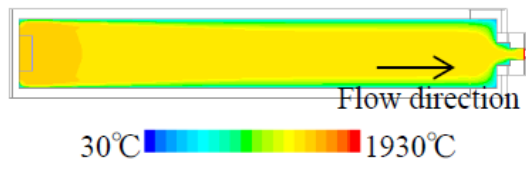

(a)Surface temperature

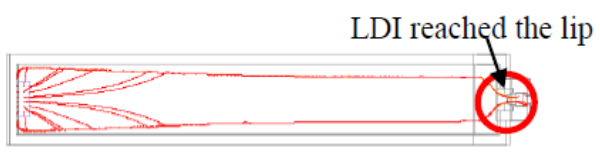

(b)Behavior of LDI

Figure 2 Behavior of LDI without IH

\begin{tabular}{|l|c|c|c|}
\hline State & Surface temperature & Behavior of LDI \\
\hline 1 & $30^{\circ} \mathrm{C}$ & \\
\hline 3 & & \\
\hline
\end{tabular}

Figure 3 Behavior of LDI with IH

\section{Verification of effectiveness of IH by experiments}

Experiments to verify the effects in actual facilities have been carried out. In the experiments, carbon tracer particles were used instead of true LDI because the density is much less than that of molten titanium. And then we mixed them with raw materials. Time transition of carbon tracer particles in molten titanium is shown in figure 4 . In the figure, the molten titanium in the cold hearth flowed from the upstream side to the downstream side, so that carbon tracer particles behaved in the same manner. In the case of a heat pattern without IH (see figure 4(a)), the particle was not eliminated and flowed to a mold. On the other hand, in the case of a heat pattern with IH (see figure 4(b)), the particle got stuck in front of the IH area and was not able to flow to the mold. Therefore, the effects of Intensive Heating on eliminating LDI have been confirmed. 


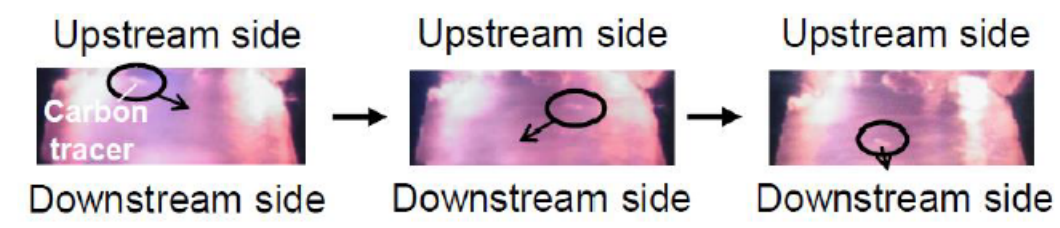

(a)Behavior of a carbon tracer particle without $\mathrm{IH}$

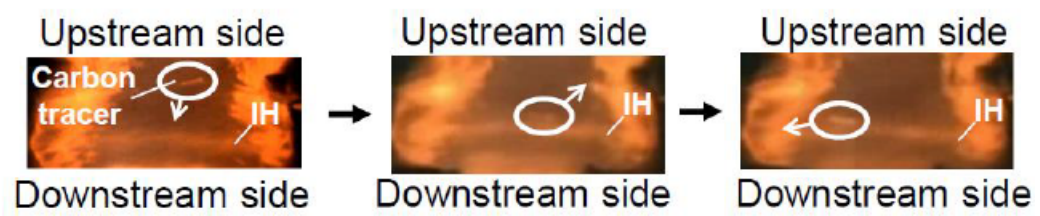

(b)Behavior of a carbon tracer particle with $\mathrm{IH}$

Fig. 4 Experimental Results to verify the effect of Intensive Heating

\section{Conclusion}

Titanium alloys used for rotating parts of aeroplanes must meet strict standards of quality, so that it is required to demonstrate that electron beam melting is capable of producing defects-free ingots. However, complete elimination by dissolution of LDI having high nitrogen content would be difficult, so that a new way of eliminating LDI was needed. Therefore, control of a state of the molten titanium flow with the aide of Marangoni flow induced by Intensive Heating has been studied and effects on eliminating LDI has been verified by the numerical simulations and the experiments using actual electron beam facility and carbon tracer particles.

\section{Acknowledgments}

The authors say thank you all co-workers for their support, encouragement and advices.

\section{References}

[1] A. Mitchell, Materials Science and Engineering A. 263 (1999) 217-223.

[2] J.P.Bellot, B.Foster, S.Hans, E.Hess, D.Ablitzer and A.Mitchell, Metallurgical and Materials Transactions B, 28B (1997) 1001-1010.

[3] J.P.Bellot, E.Hess and D.Ablitzer, Metallurgical and Maerials Transactions B, 31B (2000) 845-854.

[4] J.P.Bellot, V.Descotes and A.Jardy, Numerical Modling, 65 (2013) 1164-1172.

[5] X.Zhao, Master's thesis of the University of British Columbia, (2006).

[6] Z.Zhang, Master's thesis of the University of British Columbia, (2013).

[7] S.A.Morsi and A.J.Alexandar, J.Fluid Mech., 55 (1972) 193-208.

\section{Appendix A Properties of Ti-6Al-4V}


Properties of Ti-6Al-4V using in the numerical simulations (see section 3) are shown in table 2 . Vi assumed to be described in the polynomial form: $\mu=0.03856-2.85243 \cdot 10^{-5} \mathrm{~T}+5.45115 \cdot 10^{-9} \mathrm{~T}^{2}$.

Table 2 Properties of Ti-6Al-4V

\begin{tabular}{|c|c|c|}
\hline Temperature $[\mathrm{K}]$ & Specific heat $[\mathrm{J} / \mathrm{kgK}]$ & Thermal conductivity [W/mK] \\
\hline 298 & 546 & 7.0 \\
\hline 373 & 562 & 7.45 \\
\hline 473 & 584 & 8.75 \\
\hline 573 & 606 & 10.15 \\
\hline 673 & 629 & 11.35 \\
\hline 773 & 651 & 12.6 \\
\hline 873 & 673 & 14.2 \\
\hline 973 & 694 & 15.5 \\
\hline 1073 & 714 & 17.8 \\
\hline 1173 & 734 & 20.2 \\
\hline 1267 & 753 & 22.7 \\
\hline 1269 & 641 & 19.3 \\
\hline 1373 & 660 & 21.0 \\
\hline 1473 & 678 & 22.9 \\
\hline 1573 & 696 & 23.7 \\
\hline 1673 & 714 & 24.6 \\
\hline 1773 & 732 & 25.8 \\
\hline 1873 & 750 & 27.0 \\
\hline 1922 & 759 & 28.4 \\
\hline 1924 & 831 & 33.4 \\
\hline
\end{tabular}

